\title{
Rhabdomyomatous mesenchymal hamartoma developed at an unexpected location
}

\author{
Ali Sayan ${ }^{a}$, M.D., Gulden Diniz ${ }^{b}$, M.D., PhD, Ass. Prof, Mehmet Mert , M.D., Zubeyde Yildirim Ekinc, M.D., PhD \\ and Gokhan Koyluoglud, M.D., Prof.
}

\begin{abstract}
Rhabdomyomatous mesenchymal hamartoma is a rare dermal lesion which was first described in 1986 as "striated muscle hamartoma". It usually develops in the head and neck region of newborns.

We report a 38-day-old girl with a congenital skin tag in the perianal region. Physical examination did not reveal any congenital abnormalities or other dermal lesions. Histopathological examination showed a hamartoma with disorganized skeletal muscle fibers.

The differential diagnosis includes skin tag, accessory tragus and soft fibroma. Rhabdomyomatous mesenchymal hamartoma differs from the listed lesions with its striated muscle component. Since it does not carry the risk of recurrence and malignant transformation, it is not very important to distinguish it from these lesions. However, a correct diagnosis is important because approximately one third of the cases are associated with congenital anomalies. Also, histopathological diagnosis should be made in children with perianal localization due to similar clinical manifestation of rhabdomyosarcoma.
\end{abstract}

Key words: hamartoma, rhabdomyomatous, mesenchymal, child.

http:/ / dx.doi.org/10.5546/aap.2019.eng.e519

To cite: Sayan A, Diniz G, Mert M, Yildirin Ekin Z, Koyluoglu G. Rhabdomyomatous mesenchymal hamartoma developed at an unexpected location. Arch Argent Pediatr 2019;117(5):e519-e522.

\section{INTRODUCTION}

Rhabdomyomatous mesenchymal hamartoma (RMH) was firstly described in 1986 by Hendrick as "striated muscle hamartoma". ${ }^{1}$

a Pediatric Surgery Clinics. Izmir Tepecik Education and Research Hospital, Izmir, Turkey.

b. Pathology Department. Izmir Democracy University.

c. Pathology Department. Izmir Tepecik Education and Research Hospital, Izmir, Turkey.

d. Pediatric Surgery Clinics. Izmir Katip Celebi University.

E-mail address:

Gulden Diniz, MD, PhD: gulden.diniz@idu.edu.tr

Funding: None.

Conflict of interest: None.

Received: 9-30-2018

Accepted: 3-25-2019
The lesions are most frequently congenital, and localized in midline structures, and head and neck region. Since this lesion also contains folliculo sebaceous component, it is also called as "congenital midline hamartoma", and "skin tag hamartoma". ${ }^{2}$ In the subsequent years, since the lesion has hamartomatous characteristics, and its most discriminative feature is its striated muscle component, Mills designated it as "rhabdomyomatous mesenchymal hamartoma of the skin". ${ }^{2,3}$ The lesion has a benign nature, and it is generally detected during the neonatal period. Most $(88.2 \%)$ of the reported lesions have been localized on the head and neck region, and $26.4 \%$ of them on the jaw. However, it has been detected on tongue, and orbita. ${ }^{4-6} \mathrm{RMHs}$ may be very frequently accompanied by congenital anomalies. ${ }^{2}$

In our study we present an atypically localized case of RMH which was previously reported only once by Sampat et al., ${ }^{7}$ in the year 2017. Although any other congenital anomaly was not associated with $\mathrm{RMH}$, we want to attract attention to its importance in the differential diagnosis of congenital anomalies that may accompany RMHs. The differential diagnoses for $\mathrm{RMH}$ also include fetal rhabdomyoma and smooth muscle hamartoma. Perianal lesions may be confused with other malignant lesions as rhabdomyosarcoma (RMS) or Langerhans-cell histiocytosis (LCH). However RMS and LCH immunohistochemically are different from $\mathrm{RMH} .^{1-3}$

\section{CASE PRESENTATION}

A 38-day $3200 \mathrm{~g}$ baby girl born at term with normal spontaneous delivery was brought into our clinic with complaints of a congenital perianal skin tag which was reportedly grown day by day. On her physical examination, any additional congenital anomaly or any other similar lesion was not detected. Her motor and mental development was in concordance with her age. Physical examination of the patient in the lithotomy position revealed two skin tags, one measuring nearly $1.5 \mathrm{~cm}$ at $3 \mathrm{o}^{\prime}$ clock position lateral to the anus, and the other one measuring approximately $0.5 \mathrm{~cm}$ at 7 o' clock 
position (Figure 1). Microscopic examination of the surgically excised tissue showed randomly localized degenerated muscle fibers under intact epidermis (Figure 2). Muscle fibers stained red with Masson's trichrome dye (Figure 3). Immunohistochemical staining with sarcomeric actin demonstrated that these muscle fibers were striated muscle fibers (Figure 4). In addition, there were a lot of histiocytes which were demonstrated by anti CD68 antibody (Figure 5). This lesion rich in striated muscle component was informed as "rhabdomyomatous mesenchymal hamartoma".

\section{DISCUSSION}

Rhabdomyomatous mesenchymal hamartoma is a benign lesion with presumably hamartomatous nature. Its pathogenesis is not well established. Development of RMH has been thought to take place due to erroneous pathway of migration of tissues of mesodermal origin in embryogenesis. ${ }^{2,3}$ Its striated muscle component is thought to be constructed by fragments of superficial muscular structures which originate from the second branchial arch as m.orbicularis oculi, m.orbicularis oris or m. platysma which led an erroneous pathway of migration. ${ }^{3}$

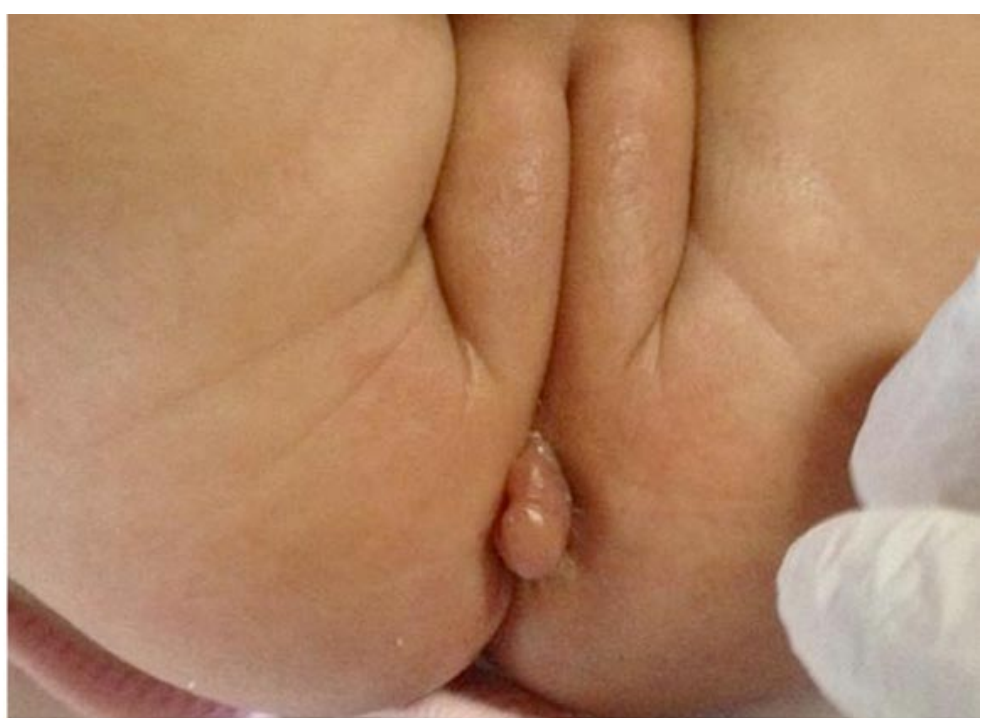

FIGURE 2. Note clusters of striated muscle fibers (arrow) in the dermis (HE X 400)

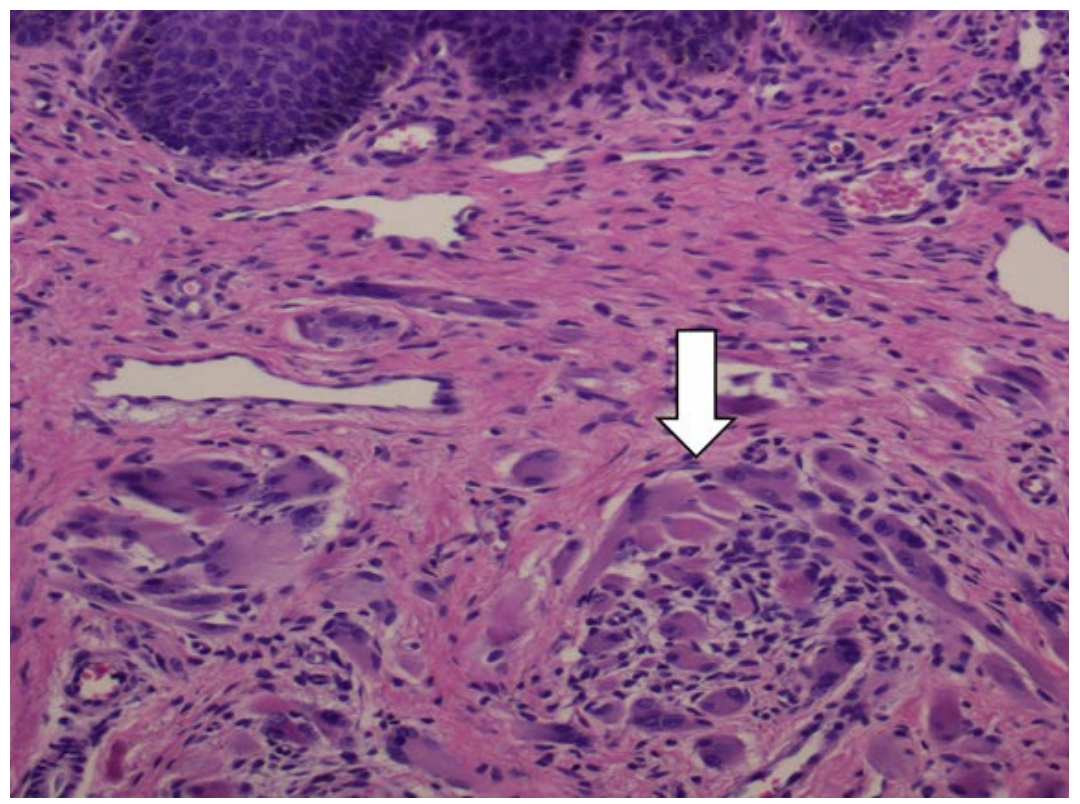


In a literature screening published by Hao et al., ${ }^{8}$ a total of 39 cases with RHM were reported. A correlation could not be established between RMH and gender, and RMH was generally observed as a solitary lesion. Generally RMH has been reported in association with congenital anomalies as cleft palate, retinal dysplasia and congenital amniotic band syndrome. ${ }^{2}$ Sanchez et al., ${ }^{9}$ reported a case of $\mathrm{RMH}$ in association with Delleman syndrome. This syndrome is also called oculocerebrocutaneous syndrome which is characterized with orbital cyst, cerebral malformation and a lesion with skin sagging. The association of RMH with auricular anomalies and pathologies originating from branchial arch as tryglossal duct cyst, cleft palate and cleft lip has been reported. This condition supports the assertion that striated muscle component of RMH originates from a branchial arch.

Most of the reported cases of RMH are localized on head and neck region. Solis-Coria et al., ${ }^{2}$ reported sternoclavicular region as its atypical location. Scrivener et al., ${ }^{10}$ reported that polypoid lesion formed following regression of

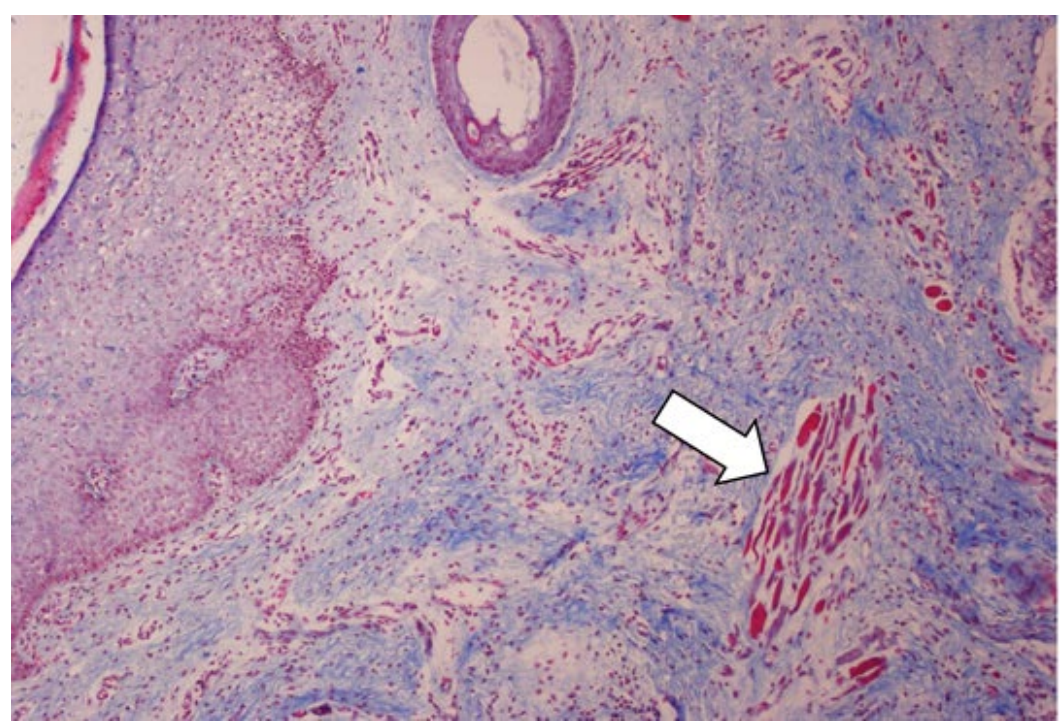

FIGURE 4. Presence of diffuse sarcomeric actin expression in striated muscle fibers (DABX 200)

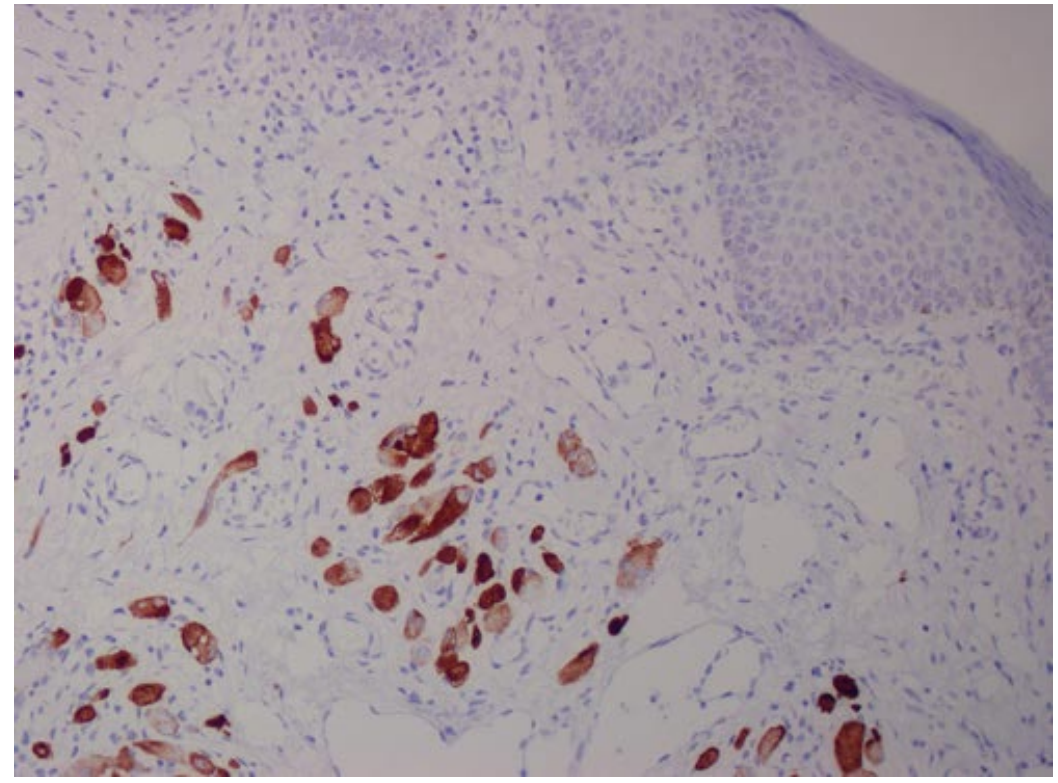




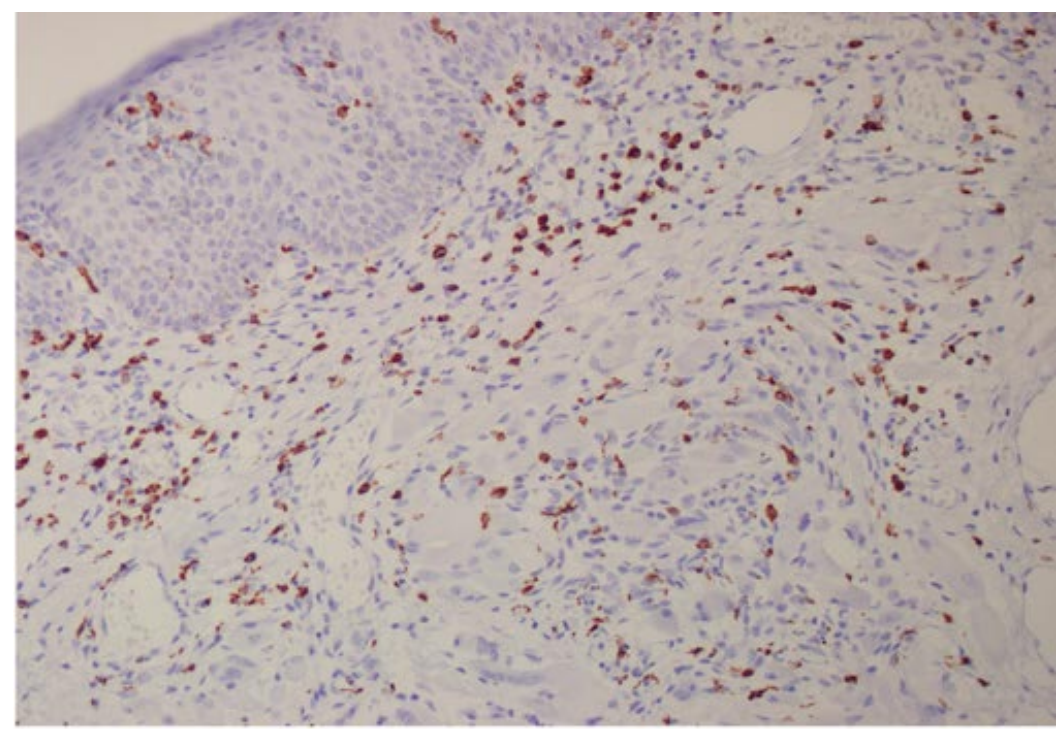

a perianal hamartoma had the characteristics of a striated muscle hamartoma. The first case of perianal RMH reported by Sampat et al., ${ }^{7}$ and any concomitant pathology was not indicated. In our presented case, any congenital anomaly or similar skin lesion was not detected. Histopathological examination of this perianal lesion revealed the diagnosis of RMH. With this presentation, we want to demonstrate that $\mathrm{RMH}$ resembles many other benign hamartomatous lesions both macroscopically and microscopically, and in cases where striated muscle component is effaced, it may easily receive a different diagnosis.

\section{CONCLUSION}

In summary, with presentation of this atypically localized case of $\mathrm{RMH}$, we try to emphasize that making differential diagnosis of RMH not only has academic significance, but it may also reveal the absolute presence of other potentially concomitant anomalies and defects. Besides, we have drawn the attention to inclusion of rhabdomyosarcoma-like malignancies in the differential diagnosis of the lesions developed on its classical location of head and neck region. ${ }^{11}$

\section{REFERENCES}

1. Hendrick SJ, Sanchez RL, Blackwell SJ, Raimer SS. Striated muscle hamartoma: Description of two cases. Pediatr Dermatol. 1986; 3(2):153-7.

2. Solis-Coria A, Vargas-Gonzáles R, Sotelo-Avila C. Rhabdomyomatous mesenchymal hamartoma presenting as a skin tag in the sternoclavicular area. Pathol Oncol Res. 2007; 13(4):375-8.

3. Rosenberg AS, KirkJ, Morgan MB. Rhabdomyomatous mesenchymal hamartoma: an unusual dermal entity with a report of two cases and review of literature. J Cutan Pathol. 2002; 29(4):238-43.

4. Mavrikakis I, White VA, Heran M, Rootman J. Orbital mesenchymal hamartoma with rhabdomyomatous features. Br J Ophthalmol. 2007; 91(5):692-3.

5. De la Sotta P, Salomone C, González S. Rhabdomyomatous (mesenchymal) hamartoma of the tongue: Report of a case. J Oral Pathol Med. 2007; 36(1):58-9.

6. Takeyama J, Hayashi T, Sanada T, Shimanuki Y, et al. Rhabdomyomatous mesenchymal hamartoma associated with nasofrontal meningocele and dermoid cyst. J Cutan Pathol. 2005; 32(4):310-3.

7. Sampat K, Cheesman E, Siminas S. Perianal rhabdomyomatous mesenchymal hamartoma. Ann R Coll Surg Engl. 2017; 99(6):e193-5.

8. Hao J, Diao QC, Wang SP, Liang CP, Shi BJ.. Rhabdomyomatous mesenchymal hamartoma:case report and literature review. Int J Dermatol. 2015; 54(10):1183-5.

9. Sánchez RL, Raimer SS. Clinical and histologic features of striated muscle hamartoma: possible relationship to Delleman's syndrome. J Cutan Pathol. 1994; 21(1):40-6.

10. Scrivener Y, Petiau P, Rodier-Bruant C, Cribier B, et al. Perianal striated muscle hamartoma associated with hemangioma. Pediatr Dermatol. 1998; 15(4):274-6.

11. Diniz G, Akgüner M, Ortaç R. A negligible diagnosis: Rhabdomyomatous mesenchymal hamartoma. Türk Patoloji Dergisi. 2008; 24(3):190-3. 